\title{
Efficacy, safety and cost analyses in ulcerative colitis patients undergoing granulocyte and monocyte adsorption or receiving prednisolone
}

\author{
Keiichi Tominaga*, Masakazu Nakano, Mina Hoshino, Kazunari Kanke and Hideyuki Hiraishi
}

\begin{abstract}
Background: Patients with ulcerative colitis (UC) are treated with prednisolone (PSL), which causes adverse side effects. Extracorporeal granulocyte/monocyte adsorption (GMA) with an Adacolumn depletes elevated/activated myeloid lineage leucocytes as sources of inflammatory cytokines. We were interested to evaluate the efficacy, safety and the treatment cost for PSL and GMA.

Methods: Forty-one patients with active UC had achieved remission with GMA, at 1 or 2 sessions/week, up to 10 sessions ( $n=24)$ or with orally administered PSL (1 mg/kg bodyweight, $n=17)$. Clinical activity index (CAl) $\leq 4$ was considered clinical remission. Following remission, patients received 5-aminosalicylic acid (2250-3000mg/day) or sulphasalazine (4000-6000 mg/day) as maintenance therapy and were followed for 600 days. The total treatment cost was assessed based on $1 €=150$ JPY.
\end{abstract}

Results: PSL was tapered after two weeks, and discontinued when a patient achieved remission. The average time to the disappearance of at least one major UC symptom (haematochezia, diarrhoea, or abdominal discomfort) was 15.3 days in the GMA group and 12.7 days in the PSL group, while time to remission was 27.9 days in the GMA group and 27.6 days in the PSL group, CAI 0.8 and 2.0, respectively. The Kaplan-Meier plots showed similar remission maintenance rates over the 600 days follow-up period. The average medical cost was 12739.4€/patient in the GMA group and $8751.3 €$ in the PSL group $(P<0.05)$. In the GMA group, 5 transient adverse events were observed vs 10 steroid related adverse events in the PSL group $(P<0.001)$.

Conclusions: In appropriately selected patients, GMA has significant efficacy with no safety concern. The higher cost of GMA vs PSL should be compromised by good safety profile of this non-pharmacological treatment intervention.

Keywords: Ulcerative colitis, Adsorptive granulocyte and monocyte apheresis, Prednisolone, Medical cost, Treatment safety

\section{Background}

Ulcerative colitis (UC) is one of the two major phenotypes of the chronic inflammatory bowel disease (IBD); the other major phenotype of IBD is Crohn's disease [1,2]. UC afflicts millions of individuals throughout the world, causing symptoms like fever, weight loss, diarrhoea, rectal bleeding and abdominal discomfort, which impair individual's activities and quality of life. Currently, there is no permanent cure for $\mathrm{UC}$, the treatments focus on

\footnotetext{
* Correspondence: tominaga@dokkyomed.ac.jp

Department of Gastroenterology, Dokkyo Medical University, 880, Kitakobayashi, Mibu, Shimotsuga, Tochigi 321-0293, Japan
}

controlling the disease activity with pharmacologics, often at high doses over long periods of time [1,3,4]. Corticosteroids like prednisolone (PSL) have had a major role in the treatment of UC since the pioneering trials by Truelove, and colleagues several decades ago $[5,6]$. However, when used in chronic, remitting-relapsing conditions like UC, corticosteroids cause adverse side effects, steroid dependency and refractoriness [1,3]. It is known that long-term administration of corticosteroids potentially increases the development of osteoporosis, and cataracts as adverse side effects, which are more likely at higher corticosteroid doses [7-9]. Accordingly, in one major study, the incidence 
of severe adverse events was higher in the high dose PSL group as compared with the low dose group [9].

There is evolving evidence for myeloid lineage leucocytes (granulocytes and monocytes) as major factors in the immunopathogenesis of UC. Thus, patients with UC may harbour elevated peripheral neutrophils [10] in the presence of compromised lymphocytes [11-13]. Further, in UC, neutrophils show activation behavior [14] and prolonged survival [15]. Factors known to promote neutrophil survival include inflammatory cytokines [16], and paradoxically corticosteroids [17]. Indeed, neutrophil activation and prolonged survival is a feature of persistent intestinal inflammation and histological examinations of the mucosal tissue in biopsy specimens from patients with active UC reveals a spectrum of pathologic manifestations among which presence of neutrophils accounts not only for the morphologic lesions of UC, but also for the prevailing patterns of mucosal inflammation $[1,14,15,18]$. When activated, granulocytes together with monocytes/macrophages produce an array of pleiotropic cytokines like tumour necrosis factor (TNF)- $\alpha$, interleukin (IL)-1 $\beta$, IL6, IL-12, IL-23 which are strongly inflammatory [19-23]. These have given rise to the inference that elevated and activated granulocytes and monocytes should be targets of therapy in UC. Based on theses observations, the Adacolumn has been developed for selective depletion of granulocytes and monocytes by adsorption (GMA).

In the early 2000, following a major multicentre clinical trial [24,25], GMA was introduced into the Japan national health reimbursement scheme as remission induction therapy for patients with UC. Subsequently, investigators reported high remission rates and good safety profile for GMA [26-30], but disappointing trial outcomes have been reported as well [31]. In this study, we aimed to assess the efficacy, safety and treatment cost in two cohorts of patients with active UC treated with GMA or with PSL as remission induction therapy.

\section{Methods}

\section{Patients}

The subjects were 41 patients with $\mathrm{UC}$ in the active stage who had been successfully treated by GMA as remission induction therapy in the period from April 2000 to March 2009 at our hospital (GMA group, $n=24$ ) or with the corticosteroid, prednisolone (PSL group, $n=17$ ). The demographic features of the included patients are presented in Table 1 . These 41 patients were retrospectively reviewed. The patients were from two bigger cohorts, 43 and 24, respectively who had responded to these interventions. The remaining patients (19 and 7) either had not achieved remission or were not eligible for this follow-up study (receiving additional medications was one exclusion criterion). Following remission induction, patients were given 5-aminosalicylic acid (5-ASA, $2250-3000 \mathrm{mg} /$ day) or sulphasalazine (4000$6000 \mathrm{mg} /$ day) as maintenance therapy. If a patient had been on azathioprine (AZA) for $>8$ weeks prior to the start of the present therapy (GMA or PSL), the patient could be included and AZA was to be continued at the same dosage if required (Table 1). PSL dose was to be tapered within two weeks following the start of administration and subsequently discontinued when patient achieved remission.

\section{Adsorptive depletion of myeloid lineage leucocytes}

Adsorptive depletion of myeloid lineage leucocytes (GMA) was done with the Adacolumn as previously described $[10,12,13,28]$. The Adacolumn is filled with cellulose acetate beads of $2 \mathrm{~mm}$ in diameter as the column adsorptive leucocytapheresis carriers [32,33]. The carriers adsorb from the blood in the column most of the granulocytes and monocytes together with a significant fraction of platelets $(\mathrm{Fc} \gamma \mathrm{R}$ and complement receptor bearing cells); lymphocytes are spared. In fact lymphocytes increase after GMA [12,13,32,33], including the CD4+CD25high+ phenotype, which is known as a key regulator of immune function [33,34]. Each patient received 1 or 2 GMA sessions/week, up to 10 sessions. One session was $60-90 \mathrm{~min}$ at $30 \mathrm{~mL} / \mathrm{min}$ [28].

\section{Treatment of patients in the PSL group}

In the PSL group, patients received PSL orally, at $1 \mathrm{mg} / \mathrm{kg}$ (bodyweight)/day orally as the starting dose. The PSL dose was to be tapered at 5 to $10 \mathrm{mg}$ per week within 2 weeks following initiation of therapy. Following remission, PSL could be discontinued and replaced with maintenance therapy, 5-ASA or sulphasalazine (indicated above).

\section{Assessment of disease activity and treatment efficacy}

UC clinical activity index (CAI) was determined at entry and at appropriate time points during active treatment and the 24 months follow-up period according to Rachmilewitz [35]. Factors considered in the present study for assessing efficacy were blood in the stool, diarrhoea and abdominal pain as major complication of UC that most seriously impact activities and quality of life $[1,2]$. The time to the cessation of at least one major UC symptom as well as the time to remission $(\mathrm{CAI} \leq 4)$ was used to assess the immediate efficacy of the treatments, while the long-term effect on UC activity was assessed by the Kaplan-Meier survival graphs. The cumulative dose of PSL, average treatment cost per patient were also determined.

\section{Ethical considerations}

In Japan, GMA with the Adacolumn is an officially approved therapeutic option for patients with active IBD. 
Table 1 Entry demography of the 41 patients of this study

\begin{tabular}{llll}
\hline & & GMA & PSL \\
\hline Gender: & Male/Female & $14 / 10$ & $7 / 10$ \\
Mean age (range) & & $33.6(15-78)$ & $35.4(13-65)$ \\
Extent of UC & Total & 17 & 10 \\
& Left sided & 6 & 5 \\
Clinical course & Recto-sigmoid & 1 & 2 \\
& Chronic continuous type & 0 & 1 \\
Inpatient/Outpatient & Relapsing remitting type & 28 & 14 \\
Patients with a history of exposure to PSL & First UC episode & 0 & 2 \\
Patients on AZA (50mg/day) & & $19 / 5$ & $17 / 0$ \\
\hline
\end{tabular}

GMA, adsorptive granulocyte and monocyte apheresis; $P S L$, predonisolone; $A Z A$, azathioprine (started $\geq 8$ weeks ahead of entry).

All patients were included following a relapse of ulcerative colitis (UC)

Additionally, informed consent was obtained from all patients after explaining the study aim and the nature of the procedures involved. Adherence was made to the Principle of Good Clinical Practice and the Declaration of Helsinki at all times.

\section{Statistical analysis}

Where appropriate, data are presented as the mean \pm $\mathrm{SD}$ values. The differences between sets of parametric data were analyzed by using the $t$-statistic. The relapse rate was estimated by the Kaplan-Meier survival graphs and the log-rank test. $\mathrm{P}<0.05$ was considered significant.

\section{Results}

Time to the disappearance of at least one major UC symptom and clinical remission

Figure 1 shows time to the disappearance of at least one major UC complication like diarrhoea, haematochezia or abdominal discomfort as well as time to remission (CAI $\leq 4$ ) in the two groups following start of interventions (GMA and PSL). As shown, no statistically significant difference between the patients who received PSL and those who received the non-pharmacologic, GMA was found. The average time to the cessation of at least one major UC complication was $15.6 \pm 15.2$ days in the GMA group and $12.7 \pm 12.9$ days in the PSL group. Likewise, the average time to clinical remission was 27.9 \pm 19.6 days in the GMA group and $27.6 \pm 29.0$ days in the PSL group.

\section{Efficacy outcomes based on CAI}

The outcomes of the efficacy assessment based on CAI is presented in Figure 2, essentially echoing the outcomes seen in Figure 1. The average CAI value when at least one of the major complications of UC disappeared was $4.3 \pm 2.9$ in the GMA group and $6.7 \pm 3.4$ in the
PSL group indicating that at this time point, clinical remission was not achieved by all patients. Instead, the CAI value was significantly reduced in both groups relative to active disease, but without any significant difference between the two groups. Similarly, the average CAI value when the patients achieved remission was $0.8 \pm$ 1.3 in the GMA group and $2.0 \pm 2.2$ in the PSL group, without significant difference between the GAM and the PSL groups.

\section{The Kaplan-Meier graphs of remission maintenance}

Figure 3 shows the Kaplan-Meier survival graphs of remission maintenance during a 600 day follow-up observation at the end of active treatment. As shown, the rate

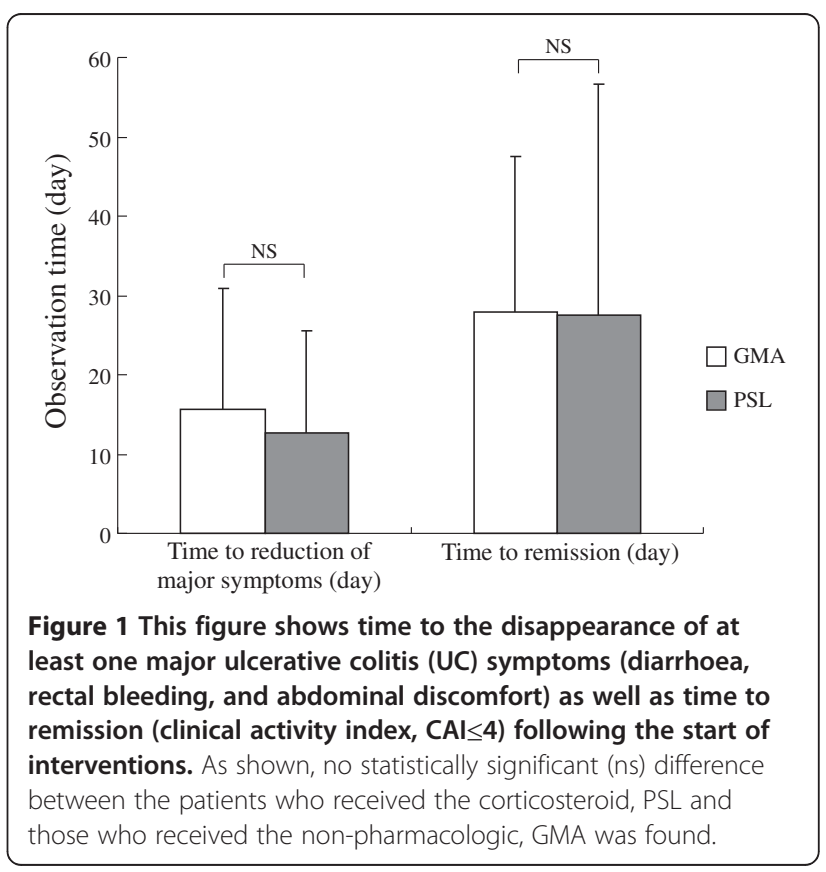




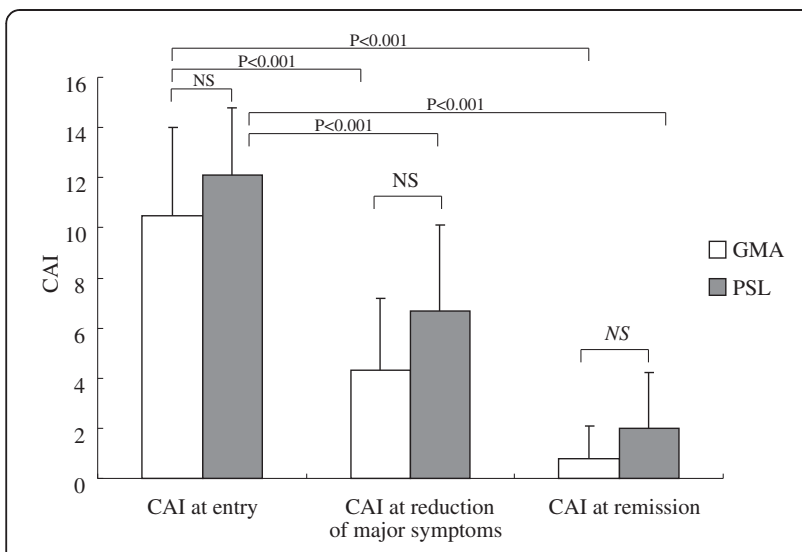

Figure 2 Overall improvements in the clinical activity indices (CAI) in two groups of patients with active UC receiving GMA or PSL. This figure shows average CAI values at baseline, at the time point when major UC symptoms were reduced and when patients achieved remission. No significant (ns) difference between the patients who received PSL and those who received the nonpharmacologic intervention, GMA was found.

of relapse was not significantly different between the GMA and the PSL groups.

\section{The cumulative dose of corticosteroid in the GMA and the} PSL groups

From patients' case records, we could calculate the total amount of corticosteroid patients had received for the duration of UC in either group. Corticosteroid was PSL in all patients at our hospital. The cumulative amount of

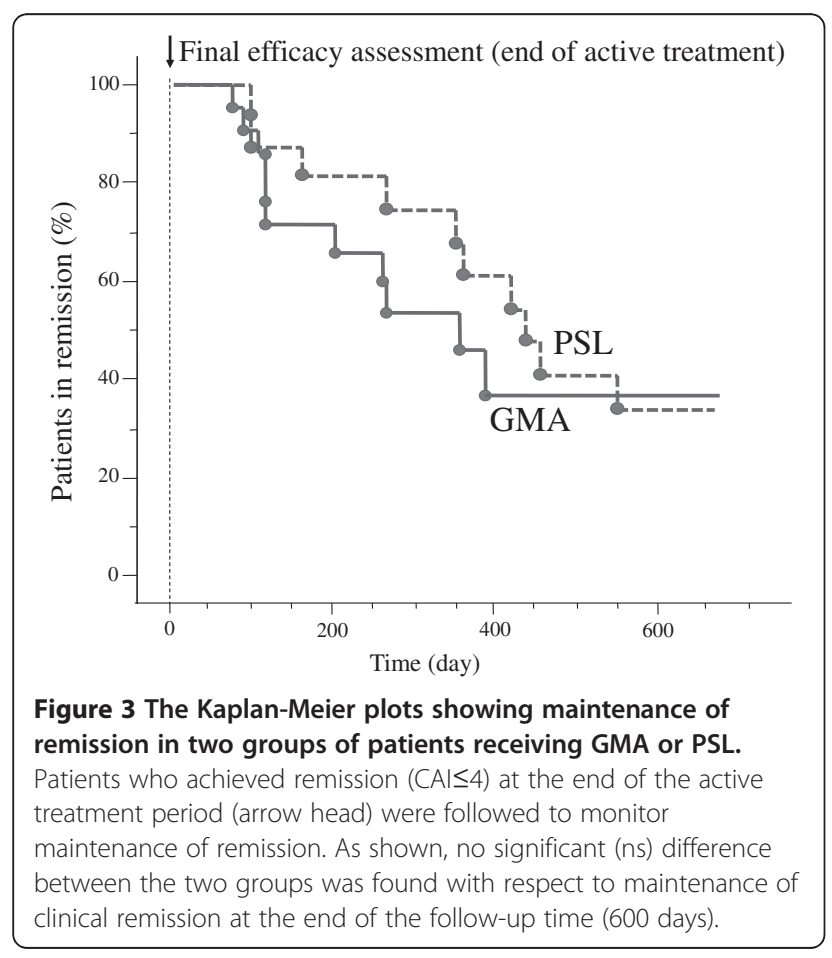

PSL before the start of the present investigation was $2556.8 \mathrm{mg}$ in the GMA group and 3030.4mg in the PSL group, not significantly different (Table 2). The cumulative amount of PSL during the present study was $0 \mathrm{mg}$ in the GMA group and $1122.4 \mathrm{mg}$ in the PSL group $(\mathrm{P}<0.001)$. All patients in the GMA group were steroid naïve for that flare up.

\section{Adverse events and treatment cost}

Figure 4 shows the outcomes of the adverse event assessment, and medical costs $(1 €=150 \mathrm{JPY})$ in the two groups of patients with UC who received GMA or PSL as a treatment intervention. Five adverse events in 3 of 24 patients $(12.5 \%)$ were reported in the GMA group. These were transient fever, dizziness and light headedness developed towards the end of a GMA session. These events remitted within $3 \mathrm{~h}$ without any medication. In the PSL group, 6 of 17 patients (35.3\%) developed corticosteroid related adverse events, total 10 events including steroid acne in 2 patients, mild liver disorder in 2 patients, moon face in 2 patients; osteoporosis in 1 patient and Candida oesophagitis in 1 patient Additionally two patients (11.8\%) in the PSL group developed nausea just after the administration of PSL. The latter two events resolved within a few hours similar to the events in the GMA group. Regarding the treatment cost in the present investigation, our calculation showed an average cost of $12739.4 €$ per patient for GMA and $8751.3 €$ per patient for PSL $(\mathrm{P}<0.05)$.

\section{Discussion}

The major focus of this study was on the safety, efficacy, and the medication cost analyses in patients with active UC being treated by GMA as a non-pharmacologic intervention (GMA group) or with the corticosteroid, PSL (PSL group). All included patients had already achieved remission following these interventions and were reviewed retrospectively. This was possible for the fact that all patients were registered at our hospital and had been treated by the physicians who are the authors of this article. Further, for the analyses we undertook in this study, patients who had achieved remission were included to allow us to see the sustainability of remission achieved with these two interventions. The outcomes might be summarised as follows. As the first treatment end point, we considered UC symptoms, which patients consider as the most serious cause of impaired activities and quality of life. These included diarrhoea, rectal bleeding, and abdominal discomfort. The average time to the cessation of at least one of these symptoms was not significantly different between the patients who received PSL and those who received GMA. The second treatment end point we considered was clinical remission. Similar to the first end point, the average time to remission was not significantly different between the 
Table 2 Outcomes of the cost analyses for two groups of patients receiving adsorptive granulocyte and monocyte apheresis (GMA) or prednisolone (PSL) at the end of the study (average values are presented)

\begin{tabular}{|c|c|c|c|}
\hline Factored item & GMA & PSL & $P$ value \\
\hline \multirow[t]{2}{*}{ Days of hospitalization } & 41.1 & 41.8 & ns \\
\hline & $(n=19)$ & $(n=17)$ & \\
\hline \multirow[t]{2}{*}{ Hospitalization cost (euro)* } & 12739.4 & 8633.6 & $\mathrm{P}<0.05$ \\
\hline & $(n=19)$ & $(n=17)$ & \\
\hline \multirow[t]{2}{*}{ Cumulative dose of PSL before the treatment (mg) } & 2457.9 & 3030.4 & ns \\
\hline & $(n=24)$ & $(n=17)$ & \\
\hline \multirow[t]{2}{*}{ Concomitant dose of PSL during this study (mg) } & 0 (naïve) & 1122.4 & $P<0.001$ \\
\hline & $(n=24)$ & $(n=17)$ & \\
\hline \multirow[t]{2}{*}{ Cumulative dose of PSL up to the end of study (mg) } & 2457.9 & 4152.8 & $P=0.28$ \\
\hline & $(n=24)$ & $(n=17)$ & \\
\hline \multicolumn{4}{|l|}{ Adverse event (\%) } \\
\hline Transient & 12.5 & 11.8 & ns \\
\hline Non-transient & 0 & 35.3 & $P<0.001$ \\
\hline
\end{tabular}

* Converted at 150 yen to the euro. Hospitalization cost includes treatment cost, medication, tests.

two groups. Further, the efficacy outcomes in terms of CAI reflected the outcomes we monitored as time to the cessation of major UC symptoms and UC remission. The sustainability of remission was another significant consideration in this study. To see this, we applied the Kaplan-Meier survival analysis of remission maintenance during a 600 day follow-up. The rate of relapse was not significantly different between the two groups.

Regarding the cumulative amount of PSL, in the GMA group, patients had relapsed while not being on a corticosteroid (were steroid naïve for that relapse). These patients received GMA as the only medication for that flare up except that AZA could be continued if a patient had started well in advance of receiving GMA. Nonetheless, we calculated the total amount of PSL patients in the two groups had received for the duration of UC disease. Our calculations did not show any significant difference in the amount of corticosteroid patients had received before the start of the medications factored into this investigation (GMA and PSL). This was because, like the patients in the GMA group, patients in the PSL group had relapsed while not being on a corticosteroid. Further, for this investigation, we ensured that all patients in the GMA group remain corticosteroid free during the follow-up time as well and in the PSL group, the dose of PSL was tapered when patients improve and discontinued when patients achieved

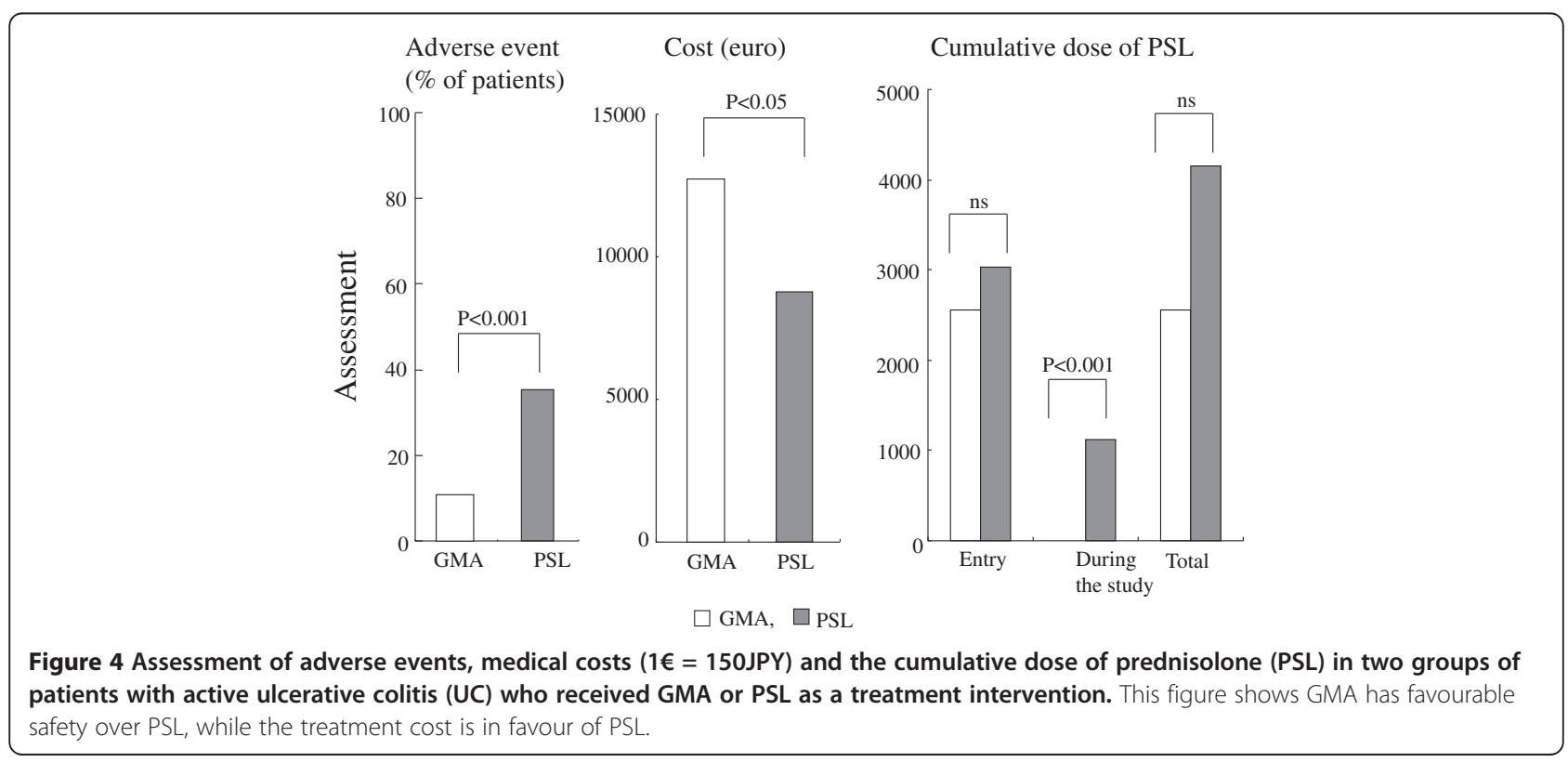


remission. Accordingly, the cumulative amount of PSL during the present study was 0mg in the GMA group and 1122.4 mg per patient in the PSL group, clearly showing that GMA spares patients from corticosteroids. Similarly, our safety evaluation showed the data to be very much in favour of GMA with just a few transient and non-serious adverse side effects, which is in line with earlier reports on the safety of GMA in patients with UC [10,12,25-33]. In contrast, over $40 \%$ of patients in the PSL group developed adverse events. However our figures from the calculations of the medication cost was in favour of PSL as this is a relatively inexpensive medication as compared with the single use Adacolumns required for GMA. Our impression is that this cost difference is more than offset by the safety of GMA [36].

Following the publication of the first clinical trial of GMA in patients with UC [24], several investigators from Japan, Europe, and the USA [12,27-30,37-43], have reported varying efficacy outcomes ranging from an impressive $85 \%$ [12] to a statistically insignificant level [31] Except $[31,37,38,41]$, most other studies did not include a control arm, relying on patients' disease activity at baseline to serve as a control parameter to judge treatment efficacy [12,27-30]. Among controlled studies, Maiden et al., used GMA to suppress clinical relapse in one arm, while the control arm received no treatment [14]. At the end of a 6-month follow-up, both the relapse rate and time to clinical relapse were significantly better in the GMA arm [14]. Further, intensive GMA involving two sessions per week was found to be more effective than the routinely applied weekly GMA sessions [44]. Likewise, there are reports saying that patients with deep colonic lesions and extensive loss of the mucosal tissue at the lesion sites show very poor response to GMA [12,29,43], while first episode and steroid naïve cases respond well and avoid corticosteroids [12,29,39].

\section{Conclusions}

The results of this study showed that GMA as a nonpharmacologic treatment intervention in patients with UC produced efficacy equivalent to the corticosteroid, PSL and was without safety concern. In view of serious adverse side effects associated with long-term administration of drugs, GMA is very much favoured by patients for its safety profile. In particular, young and elderly patients should benefit most from GMA as a replacement for corticosteroids and other potentially unsafe medications including biologics. However, the results of this study require further support by a prospective controlled study in large cohorts of patients.

\footnotetext{
Abbreviations

5-ASA: 5-aminosalicylic acid; CAl: Clinical activity index; GMA: Granulocytes and monocytes apheresis; IBD: Inflammatory bowel disease;

PSL: Prednisolone; UC: Ulcerative colitis.
}

\section{Competing interest}

The authors declare that they have no competing interests.

\section{Authors' contribution}

1) $\mathrm{KT}, \mathrm{KK}$, and $\mathrm{HH}$ : Made substantial contribution to the conception, study design and preparation of the manuscript draft; 2) KT, MN, MH: Patient management, generation, collection, assembly, analysis and interpretation of data; 3) KT, MN, MH, KK, and HH: Revision and approval of the final version of the manuscript.

\section{Acknowledgement}

No external fund was used to carry out this investigation. Further, the authors hereby declare having no conflict of interest in connection with the publication of this manuscript.

Received: 22 September 2012 Accepted: 25 February 2013

Published: 1 March 2013

\section{References}

1. Allison MC, Dhillon AP, Lewis WG, Pounder RE: Inflammatory bowel disease. London: Mosby; 1998:9-95.

2. Podolsky DK: Inflammatory bowel disease. N Engl J Med 2002, 347:417-419.

3. Present $\mathrm{DH}$ : How to do without steroids in inflammatory bowel disease. Inflamm Bowel Dis 2000, 6:48-57.

4. Hanauer SB: Medical therapy of ulcerative colitis. Gastroenterology 2004, 126:1582-1592.

5. Truelove SC, Witts LJ: Cortisone in ulcerative colitis; final report on a therapeutic trial. Br Med J 1955, 2:1041-1048.

6. Truelove SC, Jewell DP: Intensive intravenous regimens for severe attacks of ulcerative colitis. Lancet 1974, 1:1067-1070.

7. Schulte CM: Review article: bone disease in inflammatory bowel disease. Aliment Pharmacol Ther 2004, 20(Suppl 4):43-49.

8. Tripathi RC, Kipp MA, Tripathi BJ, et al: Ocular toxicity of prednisone in pediatric patients with inflammatory bowel disease. Lens Eye Toxic Res 1992, 9:469-482.

9. Saag KG, Koehnke R, Caldwell JR, et al: Low dose long-term corticosteroid therapy in rheumatoid arthritis: an analysis of serious adverse events. Am J Med 1994, 96:115-123.

10. Saniabadi AR, Hanai H, Sawada K, et al: Adacolumn, an adsorptive carrier based granulocyte and monocyte apheresis device for the treatment of inflammatory and refractory diseases associated with leukocytes. Ther Apher Dial 2003, 7:48-59.

11. Heimann TM, Aufses AH Jr: The role of peripheral lymphocytes in the prediction of recurrence in Crohn's disease. Surg Gynecol Obstet 1985, 160:295-298.

12. Suzuki Y, Yoshimura N, Saniabadi AR, Saito Y: Selective neutrophil and monocyte adsorptive apheresis as a first line treatment for steroid naïve patients with active ulcerative colitis: a prospective uncontrolled study. Dig Dis Sci 2004, 49:565-571.

13. Aoki H, Nakamura K, Yoshimatsu Y, Shirai K, Suzuki Y: Adacolumn selective leukocyte adsorption apheresis in patients with active ulcerative colitis: clinical efficacy, effects on plasma IL-8 and the expression of toll like receptor 2 on granulocytes. Dig Dis Sci 2007, 52:1427-1433.

14. McCarthy DA, Rampton DS, Liu Y-C: Peripheral blood neutrophils in inflammatory bowel disease: morphological evidence of in vivo activation in active disease. Clin Exp Immunol 1991, 86:489-493.

15. Brannigan $A E, O^{\prime}$ Connell $P R$, Hurley $\mathrm{H}$ : Neutrophil apoptosis is delayed in patients with inflammatory bowel disease. Shock 2000, 13:361-366.

16. Lee A, Whyte M, Haslett C: Inhibition of apoptosis and prolongation of neutrophil functional longevity by inflammatory mediators. J Leukoc Biol 1993, 54:283-288.

17. Meagher LC, Cousin JM, Seckl JR, Haslett C: Opposing effects of glucocorticoids on the rate of appoptosis in neutrophilic and eosinophilic granulocytes. J Immunol 1996, 156:4422-4428.

18. Tibble JA, Sigthorsson G, Bridger D, Fagerhol MK, Bjarnason I: Surrogate markers of intestinal inflammation are predictive of relapse in patients with inflammatory bowel disease. Gastroenterology 2000, 119:15-22.

19. Schreiber S, Nikolaus S, Hampe J: Tumour necrosis factor alpha and interleukin 1beta in relapse of Crohn's disease. Lancet 1999, 353:459-461.

20. Papadakis KA, Targan SR: Role of cytokines in the pathogenesis of inflammatory bowel disease. Annu Rev Med 2000, 51:289-298. 
21. Cassatella MA: The production of cytokines by polymorphonuclear neutrophils. Immunol Today 1995, 16:21-26.

22. Nikolaus S, Bauditz J, Gionchetti P: Increased secretion of proinflammatory cytokines by circulating polymorphonuclear neutrophils and regulation by interleukin-10 during intestinal inflammation. Gut 1998, 42:470-476.

23. Neurath MF: IL-23: a master regulator in Crohn's disease. Nat Med 2007, 13:26-28.

24. Shimoyama T, Sawada Y, Tanaka T, et al: Granulocyte and monocyte apheresis in the treatment of active ulcerative colitis (Engl Abstr). Jpn J Apher 1999, 18:117-131.

25. Shimoyama T, Sawada K, Kashiwagi N, Saniabadi A: Safety and efficacy of granulocyte and monocyte apheresis in patients with active ulcerative colitis: a multicenter study. J Clin Apher 2001, 16:1-9.

26. Panés J, Guilera M, Ginard D, Osa J, González-Carro P, González-Lara V, Varea $V$, Domènech E, Badia X: Treatment cost of ulcerative colitis: is apheresis with Adacolumn cost-effective? Dig Liver Dis 2007, 39:617-625.

27. Domènech E, Hinojosa J, Esteve-Comas M, Spanish Group for the Study of Crohn's Disease and Ulcerative Colitis (GETECCU), et al:

Granulocyteaphaeresis in steroid-dependent inflammatory bowel disease: a prospective, open, pilot study. Aliment Pharmacol Ther 2004 20:1347-1352.

28. Kanke $\mathrm{K}$, Nakano M, Hiraishi $\mathrm{H}$, Terano A: Clinical evaluation of granulocyte/monocyte apheresis therapy for active ulcerative colitis. Dig Liver Dis 2004, 36:811-817.

29. Tanaka T, Okanobu H, Kuga Y, et al: Clinical and endoscopic features of responders and non-responders to adsorptive leucocytapheresis: a report based on 120 patients with active ulcerative colitis. Gastroenterol 2010, 34:687-695.

30. Ljung $T$, Thomsen $\bigcirc \varnothing$, Vatn $M$, et al: Granulocyte, monocyte/macrophage apheresis for inflammatory bowel disease: the first 100 patients treated in Scandinavia. Scand J Gastroenterol 2007, 42:221-227.

31. Sands BE, Sandborn WJ, Feagan B, et al: A randomized, double-blind, sham-controlled study of granulocyte/monocyte apheresis for active ulcerative colitis. Gastroenterology 2008, 135:400-409.

32. Saniabadi AR, Hanai $H$, Sawada K, et al: Adacolumn for selective leukocytapheresis as a non-pharmacological treatment for patients with disorders of the immune system: an adjunct or an alternative to drug therapy? J Clin Apher 2005, 20:171-184.

33. Saniabadi AR, Hanai $H$, Fukunaga $K$, et al: Therapeutic Leucocytapheresis for inflammatory bowel disease. Transf Apher Sci 2007, 37:191-200.

34. Yokoyama Y, Fukunaga K, Fukuda Y, et al: Demonstration of low CD25High +CD4+ and high CD28-CD4+ T-cell subsets in patients with ulcerative colitis: modified by selective leucocytapheresis. Dig Dis Sci 2007, 52:2725-2731.

35. Rachmilewitz D: Coated mesalazine (5-aminosalicylic acid) versus sulphasalazine in the treatment of active ulcerative colitis: a randomised trial. Br Med J 1989, 298:82-86.

36. Yamamoto T: Granulocyte and monocyte adsorptive apheresis in the management of ulcerative colitis (Lttr). Aliment Pharmacol Ther 2011, 33:288.

37. Hanai H, Watanabe F, Yamada M, Saniabadi A: Adsorptive granulocyte and monocyte apheresis versus prednisolone in patients with corticosteroid dependent moderately severe ulcerative colitis. Digestion 2004, 70:36-44.

38. Hanai $H$, lida $T$, Taleuchi $K$, et al: Intensive granulocyte and monocyte adsorption versus intravenous prednisolone in patients with severe ulcerative colitis: an unblinded randomised multi-centre controlled study. Dig Liver Dis 2008, 40:433-440.

39. Tanaka T, Okanobu H, Yoshimi S, et al: Adsorptive depletion of granulocytes and monocytes impacts mucosal level of neutrophils and clinically is most effective in steroid naïve patients. Dig Liver Dis 2008, 40:731-736.

40. Caprilli R, D'Ovidio V: Leukocytapheresis as promising therapy for inflammatory bowel disease. Dig Liver Dis 2007, 39:435-437.

41. Maiden L, Takeuchi K, Bjarnason I: Selective white cell apheresis reduces relapse rates in patients with $\mathrm{IBD}$ at significant risk of clinical relapse. Inflamm Bowel Dis 2008, 14:1413-1418.

42. Bresci G, Parisi G, Mazzoni A, Scatena F, Capria A: Treatment of patients with acute ulcerative colitis: conventional corticosteroid therapy (MP) versus granulocytapheresis (GMA): a pilot study. Dig Liver Dis 2007, 39:430-434
43. Suzuki Y, Yoshimura N, Saito $Y$, Saniabadi A: A retrospective search for predictors of clinical response to selective granulocyte and monocyte apheresis in patients with ulcerative colitis. Dig Dis SC 2006, 51:2031-2038.

44. Sakuraba A, Motoya S, Watanabe K, et al: An open-label prospective randomized multicenter study shows very rapid remission of ulcerative colitis by intensive granulocyte and monocyte adsorptive apheresis as compared with routine weekly treatment. Am J Gastroenterol 2009, 104:2990-2995.

doi:10.1186/1471-230X-13-41

Cite this article as: Tominaga et al.: Efficacy, safety and cost analyses in ulcerative colitis patients undergoing granulocyte and monocyte adsorption or receiving prednisolone. BMC Gastroenterology 2013 13:41.

\section{Submit your next manuscript to BioMed Central and take full advantage of:}

- Convenient online submission

- Thorough peer review

- No space constraints or color figure charges

- Immediate publication on acceptance

- Inclusion in PubMed, CAS, Scopus and Google Scholar

- Research which is freely available for redistribution 